\title{
Utilizing Syndromic Surveillance for Hurricane Irma-Related CO Poisonings in Florida
}

\author{
Prakash R. Mulay, Kirtana Ramadugu, David Atrubin, Heather Rubino, Carina Blackmore
}

Florida Department of Health, Tallahassee, Florida, United States

Objective

This study describes how Florida Poison Information Center Network (FPICN) and emergency department (ED) data accessed through Florida's syndromic surveillance system were used to conduct near real-time carbon monoxide (CO) poisoning surveillance and active case finding in response to Hurricane Irma in Florida.

\section{Introduction}

On September 10, 2017, Hurricane Irma made landfall in Florida. Over 90\% of Florida counties reported power outages as of September 11. During power outages, CO poisonings often occur due to indoor use of fuel combustion sources (e.g., cooking, heating) or generators for electricity.

$\mathrm{CO}$ poisoning is a reportable condition in Florida; health care providers and laboratories are required to report suspected cases to the Florida Department of Health (FDOH). In Florida, approximately 202 cases of $\mathrm{CO}$ poisoning are reported each year (three-year average from 2014 to 2016). In addition to passive surveillance, FDOH uses the Electronic Surveillance System for the Early Notification of Community-based Epidemics (ESSENCE-FL) to find cases of CO poisoning.

ESSENCE-FL provides access to ED data from 98\% (255 out of 260) of EDs in Florida and all statewide FPICN call data (includes three poison control centers). ESSENCE-FL provides near real-time access to these data sets, as ED data are uploaded every 2 hours or once a day (depending on the hospital system) and FPICN data are uploaded every 10 minutes. The statewide FPICN database includes information about substance, signs and symptoms, exposure scenario, and patient identification information provided by the individual caller or clinician from a health care facility.

\section{Methods}

In addition to receipt of health care provider reports through traditional disease reporting, active case finding was conducted using ESSENCE-FL during Hurricane Irma. Exposure calls to the FPICN indicating CO exposure were extracted from the statewide database. Calls coded with the following medical outcomes were excluded: no health effect, not followed - judged as nontoxic exposure, not followed - minimal clinical effects possible, unrelated effect - the exposure was probably not responsible for the effect(s), and confirmed non-exposure. To query ESSENCE-FL ED data, a free-text query was created and executed against the concatenated chief complaint and discharge diagnosis (CCDD) field: $\left({ }^{\wedge}\right.$ carbon ${ }^{\wedge},{\text { and not },\left(,{ }^{\wedge} \text { retention }\right.}^{\wedge}$,

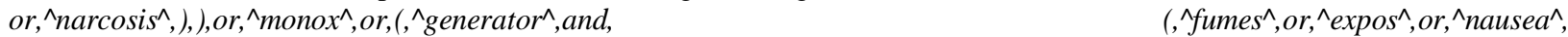
or,,$^{\wedge}$ eadach ${ }^{\wedge}$, or $^{\wedge}$ exhaust $^{\wedge}$, or,$\wedge$ garage $e^{\wedge}$, or $^{\wedge}$ inhale $\left.\left.{ }^{\wedge},\right),\right)$. Results of these queries were analyzed and sent to county and regional epidemiologists daily for investigation.

Reports of $\mathrm{CO}$ poisoning exposures were investigated by collecting medical records and conducting interviews using an expanded risk factor questionnaire [1]. Cases were classified using Florida's reportable disease case definition [2] and documented in the electronic reportable disease surveillance system, Merlin (see process flow chart). Descriptive analysis of Hurricane Irma-related $\mathrm{CO}$ poisoning cases reported in Merlin was conducted to characterize morbidity, mortality, and exposure scenarios.

\section{Results}

In September 2017, FDOH investigated 666 reports of CO poisoning and identified 529 people (79.4\%) who met the case definition for $\mathrm{CO}$ poisoning. Among 529 cases, 56.3\% were reported by ED data, 5.7\% by FPICN data, 29.1\% from both data sets, and the remaining $8.9 \%$ by other sources (e.g., self-report, media). About $60.1 \%$ of cases were only reported by FPICN and ED data, $33.1 \%$ by health care providers and laboratories, and $6.8 \%$ by other sources. Among 15 deaths, $20 \%$ were identified through active case

SDS Annual Conference Proceedings 2019. This is an Open Access article distributed under the terms of the Creative Commons AttributionNoncommercial 4.0 Unported License (http://creativecommons.org/licenses/by-nc/3.0/), permitting all non-commercial use, distribution, and reproduction in any medium, provided the original work is properly cited. 
finding using ED and FPICN data. CO poisoning cases peaked on September 12 (within two days of hurricane landfall) and decreased by September 16, as power was restored. About $95 \%$ of cases reported CO exposures within the first week of hurricane landfall.

Merlin data analysis of 529 cases identified some notable findings related to Hurricane Irma. CO poisoning rates were highest among those aged 5-14 years (4.8 per 100,000 population), and the mean age was 33.2 years (median: 31 years, range: 3 months - 89 years). Most cases were in females $(55.6 \%)$, non-Hispanics $(58.3 \%)$, and whites $(73 \%)$. CO exposures were predominantly caused by generator use (97.5\%). Among 516 generator-related exposures, $15.7 \%$ of people had a CO detector, $62.8 \%$ did not have $\mathrm{CO}$ detector, and it was unknown for $21.5 \%$. Among 516 residential exposures due to generator use, $31.3 \%$ of people reported generator use inside the home, attached garage, or other attached structures, and $66 \%$ reported generator use outside the home, including covered decks and carports. Among 340 people who reported generator use outside the home, $63.5 \%$ reported having a generator within 20 feet of windows, doors, air conditioners, or air intake vents.

\section{Conclusions}

Even though $\mathrm{CO}$ poisoning is a reportable condition in Florida, use of active surveillance was key in the public health response to Hurricane Irma- related CO poisonings. FDOH would not have identified $60 \%$ of these hurricane-related CO poisoning cases without access to FPICN and ED data. During Hurricane Irma, active case finding complemented routine disease surveillance not only in early detection of CO poisonings but also in guiding rapid public health response. Similarly, in the 2005 hurricane season, FDOH monitored FPICN data and identified an increase in CO poisonings [3]. Based on near-real-time CO poisoning surveillance, FDOH produced daily situation reports, sent out a press release about the dangers of $\mathrm{CO}$ poisoning from generator use, prepared a YouTube video, and conducted educational outreach through social media and text alert. Other jurisdictions may benefit from use of near real-time ED and poison control center data to better understand the magnitude and characteristics of CO poisonings during power outages in their areas. Public education messages need to emphasize outdoor use of generators (at least 20 feet away from doors, windows, and air conditioners) and use of $\mathrm{CO}$ detectors.

\section{Acknowledgement}

Bureau of Epidemiology staff, Florida Department of Health.

\section{References}

1. Florida Department of Health. Carbon monoxide poisoning enhanced case report form; October 2017. Available at: www.floridahealth.gov/diseases- and-conditions/disease-reporting-and-management/diseasereporting-and-surveillance/_documents/crf-co-hurricane-irma-enhanced-surveillance.pdf

2. Florida Department of Health. Carbon monoxide poisoning case definition; 2018. Available at: www.floridahealth.gov/diseases-and-conditions/disease- reporting-and-management/disease-reporting-andsurveillance/_documents/cd-carbon-monoxide.pdf

3. Monitoring Poison Control Center Data to Detect Health Hazards During Hurricane Season-Florida, 20032005. 2006. JAMA. 295(21), 2469-70. https://doi.org/10.1001/jama.295.21.2469 
Process flow for carbon monoxide surveillance during Hurricane Ima in Florida

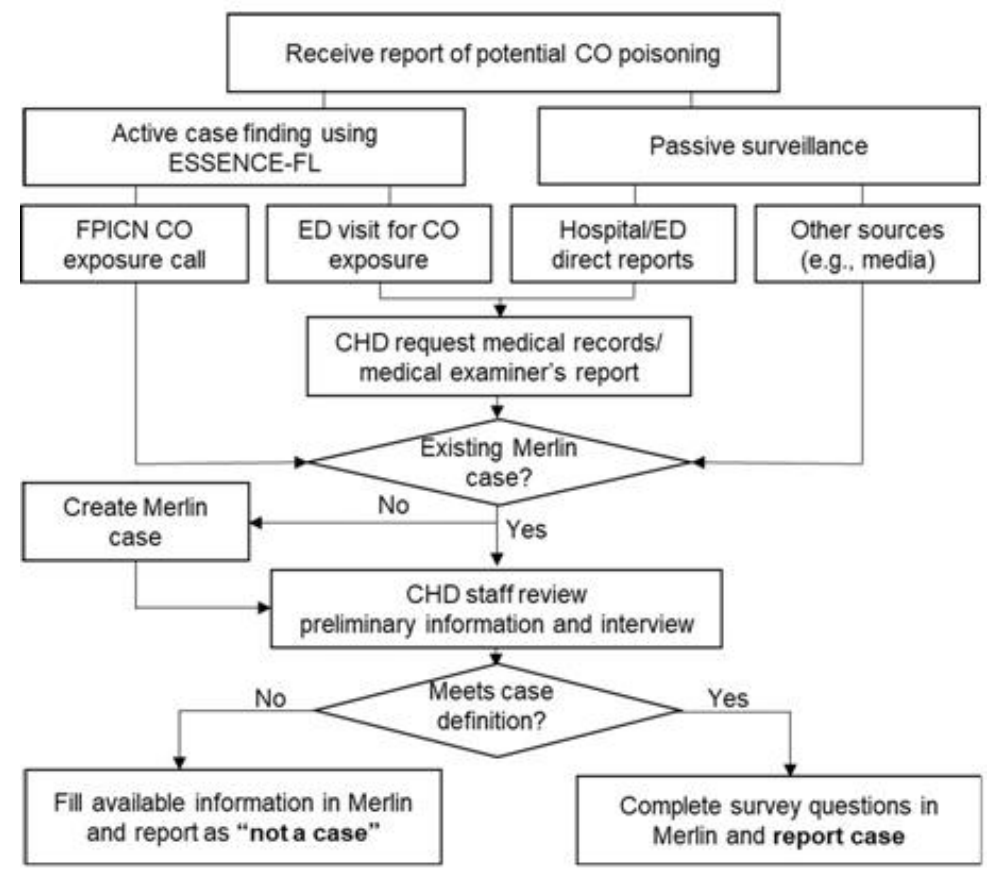

CO: Carbon Monoxide

FPICN: Florida Poison Information Center Network

ED: Emergency Department

CHD: County Health Department 